\title{
The nature of the driving mechanism in the pulsating hybrid PG 1159 star Abell 43
}

\author{
P.-O. Quirion, G. Fontaine, and P. Brassard
}

\begin{abstract}
Département de Physique, Université de Montréal, CP 6128, Succ. Centre-Ville, Montréal, Québec H3C 3J7, Canada e-mail: [quirion; fontaine; brassard]@astro.umontreal.ca
\end{abstract}

Received 10 May 2005 / Accepted 23 May 2005

\begin{abstract}
We extend our previous pulsational stability analyses of PG 1159 stars by modeling the hybrid PG 1159 type star Abell 43. We show that the standard $\kappa$-mechanism due to the ionization of $\mathrm{C}$ and $\mathrm{O}$ in the envelope of this H-rich PG 1159 star is perfectly able to drive $g$-mode pulsations. Thus, contrary to a recent suggestion, there is no need to invoke any new or exotic mechanism to explain the pulsational instabilities observed in this particular star. Our expected instability band for $l=1$ modes extends in period from $\sim 2604 \mathrm{~s}$ to $\sim 5529 \mathrm{~s}$, which is consistent with the available photometric observations of Abell 43 . We also suggest that efforts to detect luminosity variations in its sibling NGC 7094 be pursued.
\end{abstract}

Key words. stars: white dwarfs - stars: oscillations - stars: individual: Abell 43

\section{Astrophysical context}

Abell 43 is, along with NGC 7094, HS 2324+3944 and Sh 2-68, one of the four known hybrid PG 1159 stars. PG 1159 stars are usually observed as hydrogen deficient stars, but the so-called hybrid PG 1159 objects reveal strong H Balmer lines indicative of a non-negligible hydrogen abundance in their atmospheres (Napiwotzki \& Schoenberner 1991). The latest published atmospheric parameters for Abell 43 are those of Miksa et al. (2002) where we find that $T_{\text {eff }}=110000 \mathrm{~K}, \log g=5.7$, $X(\mathrm{H})=0.35, X(\mathrm{He})=0.42$, and $X(\mathrm{C})=0.23$, the latter in units of mass fraction. Abell 43 is surrounded by a planetary nebula and is classified as a lgEH object in the Werner (1992) notation. In this paper, the term PG 1159 will include hybrid PG 1159 stars.

Herwig (2001) first showed that both partial and total depletion of hydrogen in PG 1159 type stars can be explained if the last thermal pulse of the post-AGB evolutionary phase appears either early (the so-called AGB Final Thermal Pulse or AFTP) or late (the Late (LTP) or Very Late Thermal Pulse (VLTP)). An interesting characteristic of stars that undergo AFTP evolution is that they do not pass through any Born Again phase. The last He flash occurs just as they start to heat up along the postAGB track, long before the white dwarf cooling phase. In these circumstances, the convection zone provoked by the He flash does not reach the surface, so the hydrogen present in the star is not completely burned away. It is the third dredge-up, happening later, that will mix the surface and leave it with some finite amount of H. The Herwig (2001) paper presents two AFTP models ending up as PG 1159 stars with surface mass fractions of $X(\mathrm{H})=0.17, X(\mathrm{He})=0.33, X(\mathrm{C})=0.32, X(\mathrm{O})=0.15$ and $X(\mathrm{H})=0.55, X(\mathrm{He})=0.31, X(\mathrm{C})=0.07, X(\mathrm{O})=0.04$, thus giving great credibility to this formation scenario for hybrid PG 1159 stars.

Interest in Abell 43 - a peculiar object of a peculiar class is compounded by the fact that it shows multiperiodic luminosity variations. The possible variability of Abell 43, with a main period of about $2473 \mathrm{~s}$, was first reported by Ciardullo \& Bond (1996). These authors, however, used very conservative stability criteria and hesitated to conclude that Abell 43 is most likely a variable star. For their part, Schuh et al. (2000) reported that the 5500 s period they observed was a real detection at the $99 \%$ confidence level, and considered that this variation, the longest ever observed in a PG 1159 star, had a chance of being the orbital period of a binary system. More recently, Vauclair et al. (2005) positively observed $2600 \mathrm{~s}$ and $3035 \mathrm{~s}$ luminosity variations in Abell 43 which they assigned to $g$-mode pulsations. However, they doubt the fact that those modes could be driven via the classic $\kappa$-mechanism due to carbon K-shell electrons ionization as in all other known pulsating PG 1159 stars (see, e.g., Quirion et al. 2004a). Their doubts were brought about by the use of the Dreizler et al. (1995) table, where preliminary atmosphere modeling of Abell 43 gave mass fraction ratios of $X(\mathrm{H})=0.42, X(\mathrm{He})=0.51, X(\mathrm{C})=0.05$, with $\log g=5.7$ and $T_{\text {eff }}=110000 \mathrm{~K}$. This is a very low carbon abundance and it, indeed, induces no driving when modeled. We show in this paper, by using the revised table published by Miksa et al. (2002) that doubts about the nature of the driving mechanism in Abell 43 are unfounded.

Early stability calculations (Starrfield et al. 1983, 1984, 1985; Stanghellini et al. 1991) suggested that the C/O $\kappa$-mechanism in PG 1159 stars was suffering from the so-called helium and hydrogen poisoning phenomenon. Indeed, it was 
found in those computations that the composition in the driving region had to be highly helium- and hydrogen-poor in order to be able to drive pulsation modes. The hydrogen poisoning, contrary to helium poisoning, was not considered as a real problem then since pulsating hybrid PG 1159 stars were not known at the time. However, the helium poisoning problem posed a real challenge to theory because it would require the existence of very important composition gradients between the surface (typically rich in helium in PG 1159 stars) and the driving region located deeper in the envelope. No credible explanation was ever put forward to account for such large composition gradients and, furthermore, the whole concept was in direct conflict with the idea (and subsequent observational evidence) that residual stellar winds pervade the outer layers of PG 1159 stars and tend to produce homogeneous envelopes.

The puzzle of the helium poisoning problem was solved by Saio (1996) who convincingly showed that the problem was related to inadequacies in the older Los Alamos opacity data used in the previous stability calculations. Saio (1996) demonstrated that the problem simply went away with the use of the then newly available OPAL tables. In the meantime, a first hybrid PG 1159 pulsator, HS 2324+3944, showing a substantial atmospheric abundance of hydrogen $(X(\mathrm{H}) \sim 0.2)$ was discovered by Silvotti (1996). Gautschy (1997) was then able to produce a satisfactory instability strip for models resembling HS 2324+3944 including up to $20 \%$ (mass fraction) of hydrogen in the driving region. His calculations, again based on the OPAL opacity data, revoked once and for all the former allegations of hydrogen poisoning. Finally, Quirion et al. (2004a) underwent a larger model survey of PG 1159 stars, first reproducing Saio's and Gautschy's results, and then explaining the coexistence of variable and nonvariable PG 1159 stars in the $\log g-T_{\text {eff }}$ diagram in terms of a dispersion in atmospheric parameters and in terms of a variation in surface composition from star to star. This last study (also based on the OPAL dataset) confirms that the $\kappa$-mechanism due to $\mathrm{C} / \mathrm{O}$ ionization (and working in an envelope with a uniform chemical composition) is the only mechanism needed to explain the instability properties of PG 1159 stars. We complete here the survey by modeling in the same terms as before the hybrid PG 1159 star Abell 43, the one with the highest known atmospheric hydrogen abundance. Contrary to the suggestion of Vauclair et al. (2005), we find that there is no need to invoke a new or different excitation mechanism in this particular object.

\section{Models}

To model Abell 43, we used the same approach as that presented in Quirion et al. (2004a). We assumed a homogenous composition consistent with the atmospheric composition determined by Miksa et al. (2002) down to a fractional mass depth of $\log q=\log \left(1-M(r) / M_{*}\right)=-2.9$. For that part of the equilibrium model (the envelope), we thus considered a mixture made of (in mass fractions) $X(\mathrm{H})=0.35, X(\mathrm{He})=0.42$, $X(\mathrm{C})=0.22$, and $X(\mathrm{Z})=0.01$. It should be mentioned that the assumed metallicity is in solar proportions, except for $\mathrm{C}$ and $\mathrm{O}$ which are completely absent from the metal mixture $(X(\mathrm{Z}))$. Hence, our envelope is completely free of oxygen and has an

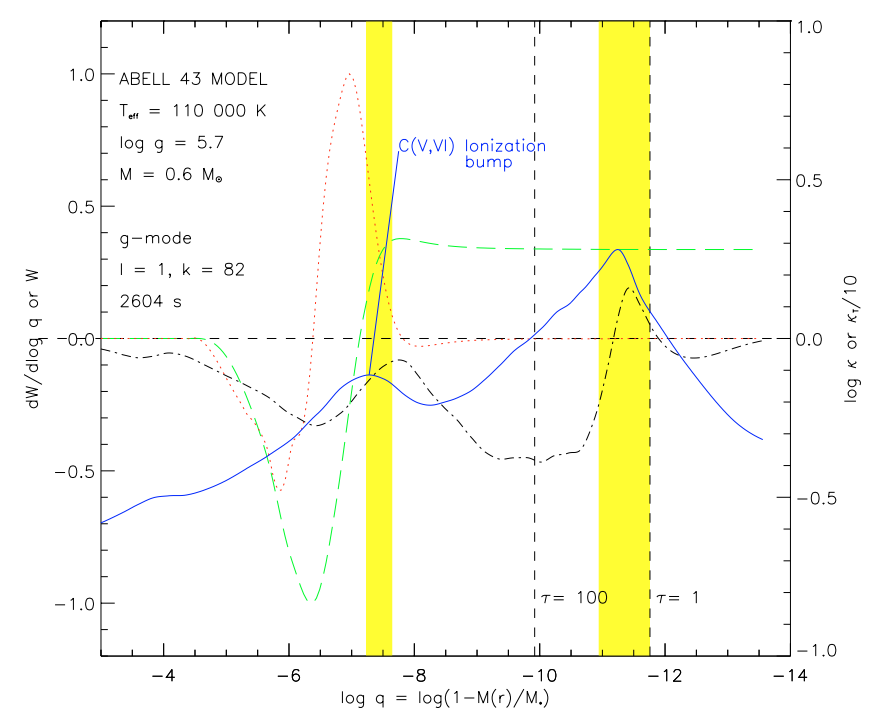

Fig. 1. Details of the driving/damping process in our model of Abell 43 for a typical excited $g$-mode. The figure shows the envelope, the part of the stellar model where the chemical composition is uniform. The location of the photosphere, $\tau_{\text {Ross }} \sim 1$, is indicated by a vertical dashed line, while the position of the base of the atmosphere, $\tau_{\text {Ross }}=100$, is indicated by a similar line. The yellow stripes give the locations and extents of two subphotospheric convection zones. The first one is just below $\tau_{\text {Ross }}=1$, and carries practically no flux, less than one tenth of a percent of the total flux. The second convection zone is linked to the $\mathrm{C}$ ionization bump and is also carrying little flux, less than $1.5 \%$. The red dotted curve refers to the derivative of the work integral $\mathrm{d} W / \mathrm{d} \log q$, and the green dashed curve to the running (from left to right) work integral $W$. Both quantities are normalized to an extremum value of either +1 or -1 . Also plotted is the run of the Rosseland opacity (blue solid curve) and the run of its logarithmic derivative $\kappa_{T}$ (black dot-dashed curve). The mode of interest has a period of $2604 \mathrm{~s}$, an e-folding time of $\sim 1300 \mathrm{yr}$, and a growth rate of $\sim 1 \times 10^{-8}$.

exact carbon mass fraction of 0.22. These details have some importance because small variations in the abundance of one of these elements can change the efficiency of the $\kappa$-mechanism, especially when a star lies near the edge of the instability strip. Note that the corresponding opacity table has been ordered on the OPAL website, which is a most useful facility. As indicated above, the effective temperature and the gravity that we used for Abell 43 are $T_{\text {eff }}=110000 \mathrm{~K}$ and $\log g=5.7$. The mass of the object, $M_{\star}=0.6 M_{\odot}$, has been chosen to be representative of a typical PG 1159 white dwarf. It has already been shown (Quirion et al. 2004b,a) that the total mass has only a small influence on the location of the instability range of PG 1159 models with similar atmospheric parameters.

The numerical tools for investigating the stability of our model were the same as those used in Quirion et al. (2004a). We carried out our search for unstable $l=1 g$-modes with periods from $1000 \mathrm{~s}$ to $6000 \mathrm{~s}$ and found instability in the range from $2604 \mathrm{~s}$ to $5529 \mathrm{~s}$. This covers rather well the range of periods detected by various authors in Abell 43 as mentioned above. We show in Fig. 1 some important characteristics of our Abell 43 model. The transition from the homogenous envelope to the $\mathrm{C} / \mathrm{O}$ core of the model has been put at $\log q=-2.9$, deep enough to have no significant influence on the driving/damping 
region located well above $\log q \simeq-4$. The only influence this deep transition zone could have on the stability analysis is a small "accordion effect" on the boundaries of the instability range as described in Quirion et al. (2004a). Essentially all the driving and damping is confined to a region of the stellar envelope associated with the bump in the opacity profile caused by $\mathrm{CV}$ and CVI ionization. This is well illustrated by the red curve in Fig. 1 which shows the typical profile of the derivative of the work integral, $\mathrm{d} W / \mathrm{d} \log q$, for a mode driven by the $\kappa$ mechanism. Our present results, along with our previous ones, demonstrate beyond any doubt that the standard $\kappa$-mechanism associated with $\mathrm{C}$ (and/or $\mathrm{O}$ ) ionization in modern models of PG 1159 stars is sufficient to explain the pulsational instabilities seen in these stars, even those of the H-rich, hybrid spectral type.

We extended, in a natural way, this study to NGC 7094, the last hybrid PG 1159 star with known and reliable atmospheric parameters which has not been modeled yet. According to Miksa et al. (2002), NGC 7094 has the same parameters as Abell 43 except for a slight variation in atmospheric chemical composition: $1 \%$ less $\mathrm{He}$ by mass that is replaced by $1 \%$ $\mathrm{O}$. Knowing that the $\mathrm{K}$-shell ionizations of $\mathrm{C}$ and $\mathrm{O}$ take place essentially in the same region of our stellar model, it was reasonable to expect that the driving mechanism would still be present with only very minor changes. And indeed, we found that, in our NGC 7094 model, pulsational instabilities of $l=1$ $g$-modes are present in the period range $2550-5413 \mathrm{~s}$. Thus, the effect of adding some trace of oxygen is to shift the band of unstable periods to slightly lower values compared to Abell 43, but the differences are quite small, as expected. In the light of this result, we strongly suggest that the 1996 efforts, undergone by Ciardullo \& Bond (1996) to detect pulsations in NGC 7094, be continued.

\section{Conclusion}

Being peculiar is the norm for the PG 1159 stars. They differ in atmospheric chemical composition, they cover a vast region in effective temperature-surface gravity space, some have a planetary nebula, and some show pulsational instabilities while others do not. Their location in the H-R diagram is one of the few places where we expect similar stars to have different looks. However, those differences in look should not overshadow the fact that those stars are all subdued to the same mechanisms. In the specific case of the pulsating PG 1159 stars, there is an unifying theme, and that is the fact that all of them excite pulsation modes through the same $\kappa$-mechanism associated with the partial ionization of $\mathrm{C}$ and/or $\mathrm{O}$ in their envelope. There is no need to invoke any new or exotic driving mechanim, even for those H-rich pulsating PG 1159 stars of the hybrid type.

This study completes the work of Quirion et al. (2004a) in that we have now modeled all PG 1159 stars with available reliable values of their atmospheric parameters and studied their pulsational stability. We have offered a consistent account of these stars, including the nonpulsators as well. We look forward to the results of future searches for pulsations in the hybrid PG 1159 star NGC 7094.

Acknowledgements. This work was supported in part by the Natural Sciences and Engineering Research Council of Canada and in part by the Fonds Québecois de la recherche sur la nature et les technologies (Québec). G.F. also acknowledges the contribution of the Canada Research Chair Program.

\section{References}

Ciardullo, R., \& Bond, H. E. 1996, AJ, 111, 2332

Dreizler, S., Werner, K., \& Heber, U. 1995, Lecture Notes in Physics (Berlin Springer Verlag), 443, 160

Gautschy, A. 1997, A\&A, 320, 811

Herwig, F. 2001, Ap\&SS, 275, 15

Miksa, S., Deetjen, J. L., Dreizler, S., et al. 2002, A\&A, 389, 953

Napiwotzki, R., \& Schoenberner, D. 1991, A\&A, 249, L16

Quirion, P.-O., Fontaine, G., \& Brassard, P. 2004a, ApJ, 610, 436

Quirion, P.-O., Fontaine, G., \& Brassard, P. 2004b, in ESA SP-559: SOHO 14 Helio- and Asteroseismology: Towards a Golden Future, 598

Saio, H. 1996, in Hydrogen-deficient star, ed. C. S. Jeffery \& U. Heber (San Francisco: ASP), ASP Conf. Ser., 96, 361

Schuh, S., Dreizler, S., Deetjen, J. L., Heber, U., \& Geckeler, R. D. 2000, Baltic Astron., 9, 395

Silvotti, R. 1996, A\&A, 309, L23

Stanghellini, L., Cox, A. N., \& Starrfield, S. 1991, ApJ, 383, 766

Starrfield, S. G., Cox, A. N., Hodson, S. W., \& Pesnell, W. D. 1983, ApJ, 268, L27

Starrfield, S., Cox, A. N., Kidman, R. B., \& Pesnell, W. D. 1984, ApJ, 281, 800

Starrfield, S., Cox, A. N., Kidman, R. B., \& Pensnell, W. D. 1985, ApJ, 293, L23

Vauclair, G., Solheim, J.-E., \& Østensen, R. 2005, A\&A, 433, 1097

Werner, K. 1992, Lecture Notes in Physics (Berlin: Springer Verlag), 401, 273 